\title{
退火气氛对镶嵌在 $\mathrm{SiO}_{2}$ 中 $\mathrm{Ag}$ 纳米颗粒热演变影响效应的研究
}

\author{
乔 瑜，金 腾，于盛旺，贺志勇，申艳艳 \\ (太原理工大学 表面工程研究所, 太原 030024)
}

摘 要: 将 $70 \mathrm{keV}$ 的 $\mathrm{Ag}$ 离子以 $5 \times 10^{16} \mathrm{~cm}^{-2}$ 的剂量注入到 $\mathrm{SiO}_{2}$ 基底中, 随后分别在 $400 \sim 800^{\circ} \mathrm{C}$ 的 $\mathrm{Ar}$ 、 $\mathrm{N}_{2}$ 、空气气 氛中退火, 详细研究了样品的表面形貌、光吸收特性、结构及成分随退火气氛及退火温度的变化规律。原子力显微 镜、紫外-可见分光光度计及掠入射 $X$ 射线衍射仪的测试结果显示: $\mathrm{Ar}$ 气氛退火样品中形成的 $\mathrm{Ag}$ 纳米粒子(NPs)细 小均匀, 其颗粒密度在 $700^{\circ} \mathrm{C}$ 时达到最大值, 光吸收性能最佳; $\mathrm{N}_{2}$ 气氛退火引发 $\mathrm{Ag}$ 纳米颗粒的团聚生长, 在样品近 表面形成较大的 $\mathrm{Ag} \mathrm{NPs}$, 其颗粒密度也在 $700^{\circ} \mathrm{C}$ 时达到最大值; 而空气中退火后, 由于 $\mathrm{AgO}$ 的形成、分解, 样品的 光吸收强度随退火温度升高持续下降。最后, 卢瑟福背散射研究结果表明, 样品的这些变化主要归因于 $\mathrm{Ag}$ 原子在 不同退火气氛下随退火温度的扩散行为不同。

关 键 词: 离子注入; $\mathrm{Ag}$ 纳米颗粒; 气氛效应; $\mathrm{Ag}$ 扩散

中图分类号: TQ174 文献标识码: A

\section{Effect of Annealing Atmosphere on Thermal Evolution of Ag Nanoparticles Embedded in $\mathrm{SiO}_{2}$ Thin Surface Layers}

QIAO Yu, JIN Teng, YU Sheng-Wang, HE Zhi-Yong, SHEN Yan-Yan

(Reseach Institute of Surface Engineering, Taiyuan University of Technology, Taiyuan 030024, China)

\begin{abstract}
Silver nanoparticles (NPs) were synthesized in thin surface layers of $\mathrm{SiO}_{2}$ glass by $70 \mathrm{keV}$ implantation of Ag ions at a fluence of $5 \times 10^{16} \mathrm{~cm}^{-2}$, and were then subjected to post thermal annealing in a temperature range of $400-800^{\circ} \mathrm{C}$ at different atmospheres, e.g. Ar, $\mathrm{N}_{2}$, air. The evolution of surface morphologies, optical absorption properties, as well as compositions and structures with annealing temperature in different annealing atmospheres were studied by AFM, UV-Vis spectrophotometry and GXRD. The results clearly show that uniformly distributed Ag NPs are observed in Ar ambient samples, which have high particle density and intense optical absorption at $700^{\circ} \mathrm{C}$. Similar optical properties are obtained in $\mathrm{N}_{2}$ annealed samples with larger Ag NPs. In contrast, the formation and decomposition of AgO significantly reduce optical absorption of air ambient samples. Moreover, the results of Rutherford backscattering spectroscopy reveal that the evolution of Ag particles should be ascribed to different diffusion behaviors of $\mathrm{Ag}$ atoms with variation of annealing temperatures and atmospheres.
\end{abstract}

Key words: ion implantation; Ag nanoparticles; atmosphere effects; diffusion of Ag atoms (2013-048) 
强的表面等离子体共振(surface plasmon resonance, SPR)性能, 成为制造光学数据存储、超快数据通信系 统、光学开关、光学传感器和分子探测器的优质潜 能材料 ${ }^{[1-4]}$ 。因此, 嵌有金属颗粒的玻璃引起了人们 的极大兴趣。众所周知, SPR 的光谱特性(如频率, 宽 度)是光电和非线性光学等未来许多应用的关键。近 年来, 研究者们提出了一些调控等离子体位置和宽 度的可行性方法, 并证明了 SPR 带的波长和宽度强 烈依赖于金属的类型和周围介质，以及金属的尺寸、 形状、分布和浓度 ${ }^{[5-7]}$ 。在合成金属纳米复合材料的 诸多技术中 ${ }^{[8-10]}$, 离子注入因具有独特的优势而备受 关注。注入元素浓度不受其在靶材料中的固溶度限 制, 注入元素的浓度和分布可以通过选择入射离子

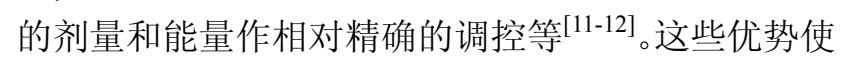
得注入的基底中产生高密度金属纳米粒子, 使合成 的金属纳米复合材料具有显著的光学效应 ${ }^{[13-14]}$ 。

$\mathrm{Ag}$ 在 $\mathrm{SiO}_{2}$ 玻璃中具有较快的迁移率 ${ }^{[16-18]}$, 在 离子注入过程中能自发团聚形成纳米粒子, 表现出 较好的 SPR 特性, 所以人们对 Ag 纳米颗粒的合成 及其结构和物性改性的研究依然不遗余力。Josep 等 ${ }^{[13]}$ 研究了 $\mathrm{KeV} 、 \mathrm{MeV}$ 级 $\mathrm{Ag}$ 离子注入 $\mathrm{SiO}_{2}$ 玻璃 基底中, 发现 $\mathrm{KeV}$ 级 $\mathrm{Ag}$ 离子注入可在基底中形成 高密度、小尺寸 $\mathrm{Ag}$ 纳米粒子, 表现出较强的 SPR 峰。任峰等 ${ }^{[15]}$ 对不同剂量 $\mathrm{Ag}$ 离子注入进行了探索, 发现低于 $10^{17}$ 剂量的注入可在 $\mathrm{SiO}_{2}$ 基底中形成颗粒 分散的球形纳米粒子。然而, 由于离子注入的特点, 离子注入合成的纳米颗粒尺寸不均匀、形状不规则。 纳米颗粒的形状、尺寸、组成和分布等又与纳米材

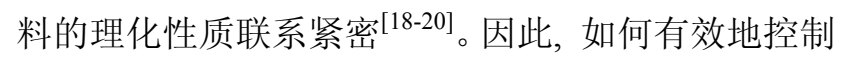
离子注入产生的纳米颗粒的尺寸、空间分布等, 进而 调控其等离子共振峰仍是目前面临的困难之一。

对离子注入后的样品进行退火处理可显著影响 注入合成的纳米颗粒结构、尺寸等, 是一种能够通 过扩散和溶质与基底缺陷的相互作用而促进粒子形 核和生长的简单、有效的方法 ${ }^{[8,15-17,21]}$ 。Ganeev 等 ${ }^{[8]}$ 指出退火可能引起基底表面纳米团簇的形成，从而 强烈地促进诸多催化反应。Roiz 等 ${ }^{[21]}$ 观察到退火温 度和气氛也影响着纳米粒子-分散纳米结构, 从而 影响材料的结构和光学性能。此外, Oliver 等 ${ }^{[16]}$ 和毕 会娟等 ${ }^{[22]}$ 分别研究了不同方法制备的 $\mathrm{Ag}$ 纳米粒子 在氧化气氛(air)和还原气氛 $\left(50 \% \mathrm{H}_{2}+50 \% \mathrm{~N}_{2}\right)$ 中的 热演变过程, 均发现在还原气氛中形成的 $\mathrm{Ag}$ 纳米 粒子具有更强的 SPR 光吸收强度。因此, 通过离子 注入法把金属嵌入绝缘体中, 并在不同条件下进行 退火处理可在一定程度上控制粒子尺寸、分布和注 入深度等。然而, 关于一定剂量 $\mathrm{Ag}$ 离子注入 $\mathrm{SiO}_{2}$
基底中并在惰性气氛( $\left.\mathrm{Ar} 、 \mathrm{~N}_{2}\right)$ 中的热演变机制如何, 尚未见报道。因此, 在前人研究的基础上, 本研究将 $70 \mathrm{keV} 、 5 \times 10^{16} \mathrm{~cm}^{-2}$ 的 $\mathrm{Ag}$ 离子注入 $\mathrm{SiO}_{2}$ 玻璃中, 在 Ar、 $\mathrm{N}_{2}$ 和空气这三种气氛下退火, 探索不同退火气 氛和退火温度对 $\mathrm{Ag}$ 纳米颗粒大小、形态、分布及 光吸收性能的影响。

\section{1 实验方法}

\section{1 实验过程}

使用 $\phi 60 \mathrm{~mm} \times 1 \mathrm{~mm}$ 光学抛光的 $\mathrm{SiO}_{2}$ 玻璃作为基 底材料, 利用金属蒸汽真空弧离子注入机在室温下对 其进行 $\mathrm{Ag}$ 离子的注入。注入能量为 $70 \mathrm{keV}$, 剂量为 $5 \times 10^{16} \mathrm{~cm}^{-2}$, 电流密度为 $0.8 \mu \mathrm{A} / \mathrm{cm}^{2} 。 \mathrm{Ag}$ 离子注入后, 将样品切成 $10 \mathrm{~mm} \times 10 \mathrm{~mm}$ 的方块, 选择部分样品在 $\mathrm{Ar} 、 \mathrm{~N}_{2}$ 和空气三种气氛中分别退火保温 $1 \mathrm{~h}$, 退火温度 $400^{\circ} \mathrm{C} \sim 800^{\circ} \mathrm{C}$, 间隔温度 $100^{\circ} \mathrm{C}$, 升温速率 $10^{\circ} \mathrm{C} / \mathrm{min}$ 。

\section{2 表征手段}

采用 UV-3600 型双光束紫外-可见(UV-Vis)分光 光度计在 200 800 $\mathrm{nm}$ 波长范围内测量样品的光吸收 谱。使用 AFM5500 型原子力显微镜(AFM)观察注入 样品的表面形貌。运用 Philips $\chi$ ’ Pert X 射线衍射仪 进行掠入射 $\mathrm{X}$ 射线衍射(GXRD)检测, 分析了注入样 品的表面相组成, 入射线为 $\mathrm{Cu} K \alpha(\sim 0.154 \mathrm{~nm})$, 扫描 范围 30 70 。最后, 采用卢瑟福背散射(RBS)技术研 究基底中注入 $\mathrm{Ag}$ 的浓度分布。分析所用的离子束为 $2 \mathrm{MeV}$ 的 $\mathrm{He}$ 离子, 束斑直径 $1 \mathrm{~mm}$, 散射角 $165^{\circ}$ 。

\section{2 结果与讨论}

\section{1 原子力显微镜分析}

注入离子在介质中因热扩散有可能相互聚集而 形成小团簇以降低自由能, 当由此引起的小团簇表 面能的增加和其所处的自由能的减少达到平衡时, 在介质内可形成稳定核, 并在适宜的条件下生长成 纳米晶体。因此, 采用 AFM 扫描对样品的注入态和 经 $\mathrm{Ar} 、 \mathrm{~N}_{2}$ 和空气气氛退火后样品的表面形貌随退火 温度的变化规律进行了观察。

从图 1(a)中可以看到, 离子注入后的样品表面 有众多小突起, 是离子注入过程中浌射玻璃表层形 成的纳米粒子高于表面的部分, 即注入态中形成了 $\mathrm{Ag} \mathrm{NPs}^{[23]}$ 。但注入态样品表面粗粘, 有许多凹坑和 沟道, 这主要是注入过程中 $\mathrm{Ag}$ 离子对 $\mathrm{SiO}_{2}$ 表面的 溅射效应引起的。因为高能离子连续不断的注入基 底中, 不仅使已形成的纳米颗粒生长, 而且引入的 


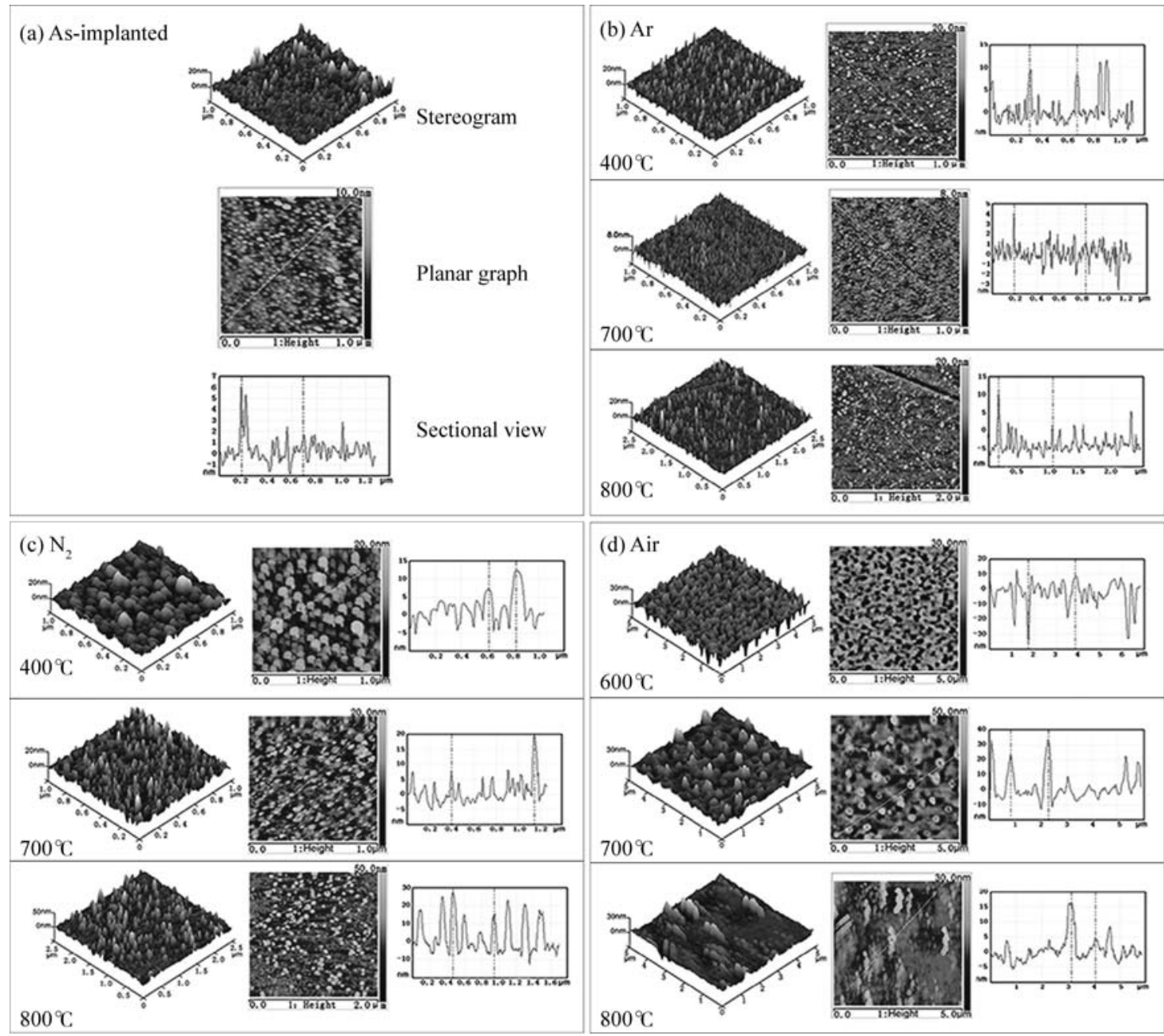

图 1 样品注入态(a)及在(b)Ar、(c) $\mathrm{N}_{2} 、(\mathrm{~d})$ 空气气氛不同温度下退火后的 AFM 图片

Fig. 1 AFM images of samples before and after annealing at elevated temperatures under different atmospheres (a) As-implanted; (b) Annealed in Ar; (c) Annealed in $\mathrm{N}_{2}$; (d) Annealed in air

辐照缺陷为纳米颗粒提供了新的有效形核位点, 导 致注入后的样品有较大的尺寸分布 ${ }^{[24]}$ 。

图 1(b)为 $\mathrm{Ar}$ 气氛中退火的样品, 由于基底温度 的升高将增加其中 $\mathrm{Ag}$ 原子的迁移率 ${ }^{[25-26]}$, 导致 $\mathrm{Ag}$ $\mathrm{NPs}$ 的形核与生长。结果 $\mathrm{Ag} \mathrm{NPs}$ 的平均高度从注入 态的 $2 \mathrm{~nm}$ 生长到 $400^{\circ} \mathrm{C}$ 退火后的 $6 \sim 10 \mathrm{~nm}$, 同时颗 粒分布的不均匀性得到了明显改善，证明离子注入 后退火能有效改变已形成纳米粒子的尺寸和分布。经 $700^{\circ} \mathrm{C}$ 退火后, 粒子平均高度降至 1 2 nm, $\mathrm{Ag} \mathrm{NPs}$ 呈细 小颗粒均匀分布于基底表面。 $800^{\circ} \mathrm{C}$ 退火后 $\mathrm{Ag} \mathrm{NPs}$ 的 平均高度略有下降 $(\sim 1 \mathrm{~nm})$, 可能表面较小的 $\mathrm{Ag}$ 纳米 团簇在高温下发生了熔融蒸发 ${ }^{[26]}$ 。图 $1(\mathrm{c}) \mathrm{N}_{2}$ 气氛中 退火时, $400^{\circ} \mathrm{C}$ 退火后粒子平均高度仅为 $4 \sim 5 \mathrm{~nm}$, $700^{\circ} \mathrm{C}$ 退火后明显增加 $(6 \sim 7 \mathrm{~nm})$ 。继续提高温度至 $800^{\circ} \mathrm{C}$ 时, $\mathrm{Ag} \mathrm{NPs}$ 生长迅速, 粒子平均高度达到 $20 \mathrm{~nm}$, 但 $\mathrm{Ag} \mathrm{NPs}$ 在 $700^{\circ} \mathrm{C}$ 时最为密集。说明 $\mathrm{N}_{2}$ 气氛中退火, 有利于 $\mathrm{Ag}$ 纳米粒子在表面生长。图 1(d)中, 经空气气氛退火后, Ag NPs 的特征随着温 度的继续升高而变得不再明显, 颗粒密度逐渐降低, 发生无序团聚现象, 样品表面一些凹坑逐步出现。 Marques 等 ${ }^{[27]}$ 发现 $\mathrm{a}-\mathrm{Al}_{2} \mathrm{O}_{3}$ 基底中注入 $\mathrm{Ag}$ 离子的样 品经氧化气氛退火后造成 $\mathrm{Ag}$ 纳米粒子偏析再结晶, 并伴随着蒸发损失。因此, 样品表面大量凹坑应该 是退火时基底内粒子运动的结果, 即退火过程中在 缺陷处聚集形成的 Ag NPs 迁移出样品表面 ${ }^{[26]}$, 留 下了缺陷形成的凹坑。而剩余的 $\mathrm{Ag}$ 原子由于热效 应聚集成团，无序地分散在基底表面。

\section{2 紫外-可见光谱分析}

图 2 给出了 $\mathrm{Ag}$ 离子注入后及样品在不同气氛 中退火的 UV-Vis 光吸收图谱。由图 2 可以看出, $\mathrm{Ag}$ 注入后的样品在 $419 \mathrm{~nm}$ 附近出现吸收峰, 为 $\mathrm{Ag}$ NPs 的 SPR 峰 ${ }^{[28]}$, 证明注入态的 $\mathrm{SiO}_{2}$ 基底中形成了 $\mathrm{Ag} N \mathrm{NP}$ 。图 2(a)所示在 $\mathrm{Ar}$ 气氛中, 由于低温退火时 大量分散的、相邻凝结核团聚形成 Ag NPs, 导致样 


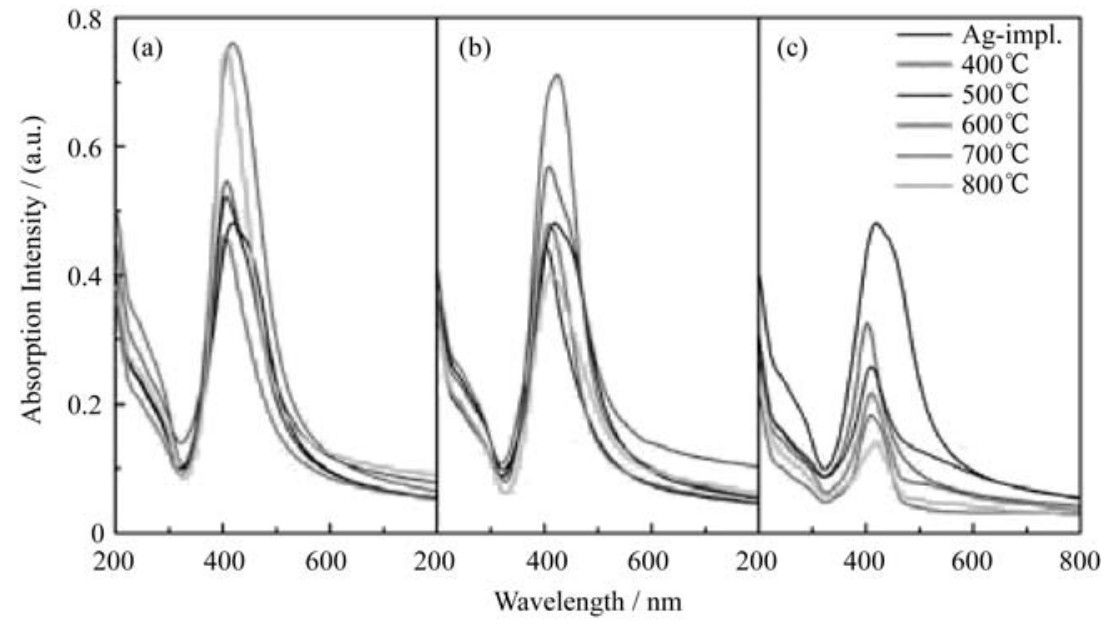

图 $2 \mathrm{Ag}$ 注入样品(a)Ar、(b) $\mathrm{N}_{2}$ 、(c) 空气气氛中退火测得的 UV-Vis 光吸收谱

Fig. 2 UV-Vis optical absorption spectra of samples before and after annealing at elevated temperatures under different atmospheres (a) Annealed in Ar; (b) Annealed in $\mathrm{N}_{2}$; (c) Annealed in air

品中 $\mathrm{Ag}$ NPs 的颗粒密度增加, 出现 $400^{\circ} \mathrm{C}$ 退火后 SPR 吸收强度的增强。因为超微颗粒的熔点远低于 其块体材料的熔点 $\left(960.8^{\circ} \mathrm{C}\right)^{[27,29]}$, 且粒子表面张力 不同, 因此在样品退火过程中, Ag NPs 可通过 Ostwald 成熟机制生长 ${ }^{[30]}$, 导致 Ag NPs 的总体密度 下降, 出现经 $500^{\circ} \mathrm{C}$ 和 $600^{\circ} \mathrm{C}$ 退火后 SPR 峰强开始下 降, 且带宽变窄。继续提高退火温度至 $700^{\circ} \mathrm{C}$ 和 $800^{\circ} \mathrm{C}$ 后, SPR 峰强急剧升高, 在 $700^{\circ} \mathrm{C}$ 达到最值, 此时注 入层中的 Ag NPs 颗粒细小均匀且具有较高的颗粒 密度(图 1(b))。 $\mathrm{N}_{2}$ 气氛中退火(图 2(b)) 的样品, 退火 温度由 $400^{\circ} \mathrm{C}$ 升高到 $700^{\circ} \mathrm{C}$ 时, 材料光吸收强度呈现 出与 $\mathrm{Ar}$ 中相似的演变趋势, 即先增后减再急剧增 强。但是经 $800^{\circ} \mathrm{C}$ 退火后, 样品内 $\mathrm{Ag} \mathrm{NPs}$ 的颗粒密 度减小(图 1(c)), 导致光吸收强度大幅度下降。然而, 空气中退火的样品(图 2(c))却表现出与 $\mathrm{Ar}$ 和 $\mathrm{N}_{2}$ 中 不同的光吸收特性。随着退火温度的升高, SPR 吸收 峰的强度持续下降。 $3 \times 10^{16} \mathrm{~cm}^{-2}$ 的 $\mathrm{Ag}$ 离子注入 $\mathrm{SiO}_{2}$ 基底中并在空气中退火后, 其光吸收谱也出现 了类似的现象 ${ }^{[16]}$ 。其原因为: 空气中退火, 由于氧 的存在, 基底表面的 $\mathrm{Ag}$ 被氧化或蒸发(图 1(d)), 降 低了 Ag NPs 的密度, 导致 SPR 峰强显著下降。

然而, 由于大多数氧化银的颗粒非常小, 无定 型, 很难在 TEM 中验证它们的存在 ${ }^{[22]}$ 。因此, 本研 究对试样进一步作了 GXRD 检测。

\section{3 掠入射 $X$ 射线衍射(GXRD)分析}

图 3 给出了样品的注入态及分别经 $A r 、 N_{2}$ 和空 气气氛不同温度退火处理后样品的 GXRD 图谱。从 图 3(a)中可以看到, 注入态在 $2 \theta$ 为 $38.17^{\circ} 、 44.11^{\circ}$ 和 $64.51^{\circ}$ 处均出现明显的衍射峰, 分别对应 $\operatorname{Ag}(111) 、 A g(200)$ 和 $\operatorname{Ag}(220)$ 晶面 ${ }^{[28]}$ 。对于 $A r$ 气氛
中退火(图 3(a))的样品, 随着温度的升高, $\operatorname{Ag}(111)$ 、 $\operatorname{Ag}(200)$ 和 $\operatorname{Ag}(220)$ 衍射峰的强度均增强, 且在 $700^{\circ} \mathrm{C}$ 退火后达到最高, 且半高宽最窄。表明此时的 $\mathrm{Ag}$ NPs 平均尺寸最大, 且有较好的结晶度。 $\mathrm{N}_{2}$ 气氛中 退火的样品中(图 3(b)), 也出现了上述的三个衍射 峰, 且衍射峰强度随退火温度的升高而增强。经 $800^{\circ} \mathrm{C}$ 退火后, $\operatorname{Ag}(111)$ 衍射峰强度达到最高, 而 $\mathrm{Ag}(200)$ 和 $\operatorname{Ag}(220)$ 峰未有明显增强, 说明 $\mathrm{N}_{2}$ 退火更有利于 $\operatorname{Ag}(111)$ 晶面的生长。如图 3(c) 所示, 样品在 $400^{\circ} \mathrm{C}$ 空气中退火后, GXRD 衍射谱在 $2 \theta=32.0^{\circ}$ 处出现新 的衍射峰, 为 $\mathrm{AgO}(200)$ 晶面衍射, 证明空气中退火, 氧气扩散进入基底中与已形成的金属团簇相互作用 形成金属氧化物 ${ }^{[31-32]}$ 。值得注意的是, 随着退火温度 升高到 $700^{\circ} \mathrm{C}, \mathrm{GXRD}$ 图谱出现多个晶化 $-\mathrm{SiO}_{2}$ 衍射 峰。其原因为: $\mathrm{Ag}$ 离子的掺杂, 降低了表层 $\mathrm{SiO}_{2}$ 的 晶化温度 ${ }^{[33]}$, 因而在氧化气氛中退火时, 注入层区 域的无定形 $\mathrm{SiO}_{2}$ 发生晶化转变。需要指出的是, 由于 $\mathrm{AgO}$ 的熔点 $\left(100^{\circ} \mathrm{C}\right)$ 要明显低于 $\mathrm{Ag}$ 的熔点 $\left(960.8^{\circ} \mathrm{C}\right)$, 因此在高温下更容易受热分解, 则经 $800^{\circ} \mathrm{C}$ 退火后, 样品中 $\mathrm{Ag}$ 含量明显减少, 只能检测到微弱的 $\operatorname{Ag}(111)$ 和 $\operatorname{Ag}(200)$ 晶面衍射峰。

\section{4 卢瑟福背散射谱分析}

图 4 给出了样品在不同气氛、不同温度退火后 测量得到的 RBS 图谱。该 RBS 图谱在 400 450 沟 道数范围内出现的峰, 对应于 $\mathrm{Ag}$ 原子引起的背散 射峰。从图中可以看到, 剂量为 $5 \times 10^{16} \mathrm{~cm}^{-2}$ 的 $\mathrm{Ag}$ 离子注入 $\mathrm{SiO}_{2}$ 玻璃后, $\mathrm{Ag}$ 元素近似呈高斯分布。图 4(a)显示, 随退火温度的升高, 大沟道数范围内 $\mathrm{Ag}$ 的背散射峰面积变化不大, 峰位向低沟道数方向略 有移动, 说明样品在 $\mathrm{Ar}$ 气氛中退火后仍保留大量 


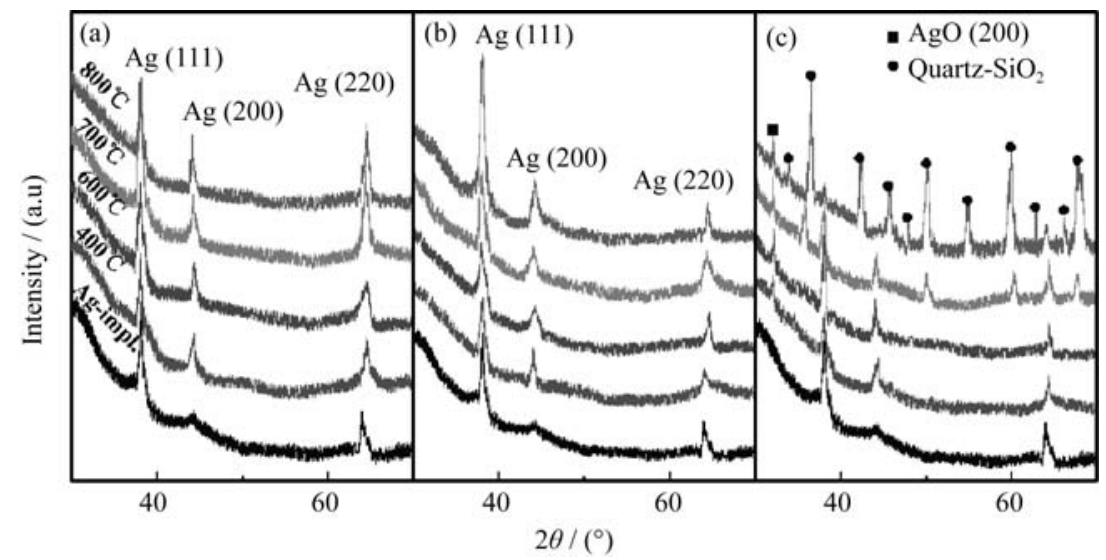

图 3 样品在(a)Ar、(b) $\mathrm{N}_{2} 、(c)$ 空气气氛中不同温度退火后得到的 GXRD 图谱

Fig. 3 GXRD patterns of samples before and after annealing at elevated temperatures under different atmospheres (a) Ar; (b) $\mathrm{N}_{2}$; (c) Air

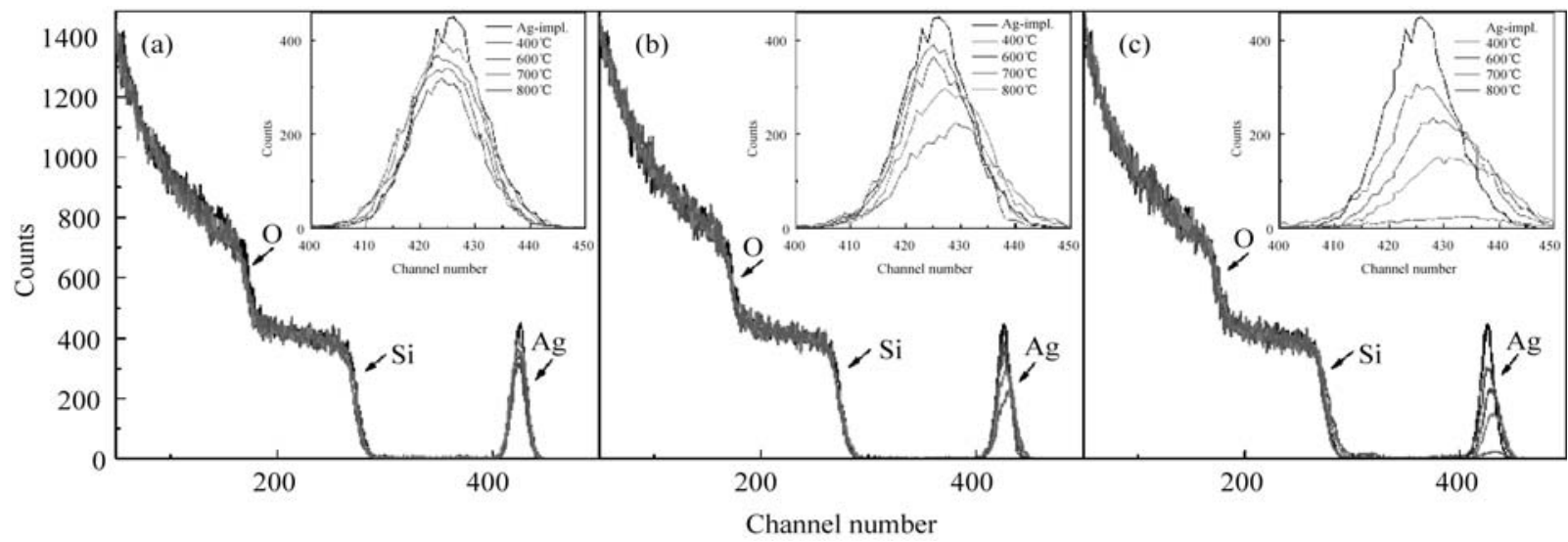

图 4 样品在(a) Ar、(b) $\mathrm{N}_{2} 、$ (c) 空气气氛中不同温度退火后得到的 RBS 图谱

Fig. 4 RBS spectra of samples before and after annealing at elevated temperatures under different atmospheres (a) Annealed in Ar; (b) Annealed in $\mathrm{N}_{2}$; (c) Annealed in air (The insets show the corresponding magnified profiles for clarity)

的 $\mathrm{Ag}$ 元素，同时 $\mathrm{Ag}$ 原子向样品深处扩散，使得 $\mathrm{Ag}$ NPs 更加稳定地嵌入 $\mathrm{SiO}_{2}$ 玻璃中, 出现 AFM 结果 所显示的基底表面 $\mathrm{Ag} \mathrm{NPs}$ 的高度随退火温度的升 高而降低的现象。图 4(b) 显示 $\mathrm{N}_{2}$ 气氛中退火时, 随 退火温度的升高, $\mathrm{Ag}$ 背散射峰空间分布区域变窄, 峰位向高沟道数方向移动较多, 表明 $\mathrm{N}_{2}$ 中退火提高 了样品中 $\mathrm{Ag}$ 原子的迁移率, 使 $\mathrm{Ag}$ 原子从最初注入 的位置向外迁移, 不仅导致 Ag NPs 在样品近表层 聚集生长形成大的 $\mathrm{Ag}$ 纳米团簇 ${ }^{[26]}$, 而且出现近表 层 $\mathrm{Ag}$ 原子受热蒸发, 明显降低了基底中 $\mathrm{Ag}$ 元素的 含量 $\left(\mathrm{Ag}\right.$ 元素的 $\mathrm{RBS}$ 产额在 $800^{\circ} \mathrm{C}$ 退火后大量减 少)。不难看出, 图 4(c)Ag NPs 在空气中退火的 RBS 图 谱具有与 $\mathrm{N}_{2}$ 中退火相似的移动规律, 可以把它归因于 空气中大量 $\mathrm{N}_{2}$ 的存在。但是, 空气中退火 $\mathrm{Ag}$ 的 RBS 产额随温度的升高显著下降, 峰面积大幅下降 ${ }^{[27]}$, 尤 其是 $800^{\circ} \mathrm{C}$ 退火后样品中仅剩余少量的 $\mathrm{Ag}$ 元素。因此, 空气退火过程中促使基底内的 $\mathrm{Ag}$ 原子具有大的向外
迁移率，且易与空气中的 $\mathrm{O}_{2}$ 分子结合形成 $\mathrm{AgO}$, 而 $\mathrm{AgO}$ 的热分解导致基底表层 $\mathrm{Ag}$ 元素蒸发损失。

\section{3 结论}

1)不同气氛中退火可以有效调控注入 $\mathrm{Ag}$ 离子 在基底表层的分布和生长状态。 $\mathrm{Ar}$ 气氛下退火促 使基底中 $\mathrm{Ag}$ 原子向基底内迁移，形成分布均匀、 颗粒细小且稳定嵌埋于 $\mathrm{SiO}_{2}$ 介质中的 $\mathrm{Ag} \mathrm{NPs}$ 。 $\mathrm{N}_{2}$ 气氛中退火则有利于基底中 $\mathrm{Ag}$ 原子向表层扩散, 引发 Ag NPs 的团聚生长, 形成较大的纳米颗粒。 而空气中退火, 由于退火过程中 $\mathrm{AgO}$ 的形成、分 解, 导致注入的 $\mathrm{Ag}$ 原子随温度的升高大量迁移出 基底表面。

2) $\mathrm{SiO}_{2}$ 介质中 $\mathrm{Ag}$ NPs 的光吸收性能与介质所处 的环境密切相关, $\mathrm{Ar}$ 气氛中经 $700{ }^{\circ} \mathrm{C}$ 退火后的 $\mathrm{Ag}$ NPs 的光吸收性能达到最佳。相比于在 $\mathrm{N}_{2}$ 和空气中 退火, $\mathrm{Ar}$ 气氛中退火有利于形成稳定嵌埋于 $\mathrm{SiO}_{2}$ 基 
底中的 Ag NPs, 从而 Ag NPs 在高温退火过程中不 易从表层扩散蒸发, 得到具有高颗粒密度的 $\mathrm{Ag}-\mathrm{SiO}_{2}$ 纳米复合材料。

\section{参考文献:}

[1] INOUYE H, TANAKA K, TANAHASHI I, et al. Ultrafast optical switching in a silver nanoparticle system. Jpn. J. Appl. Phys., 2000, 39(9A): 5132-5133.

[2] DONG P P, LIN Y Y, DENG J J, et al. Ultrathin gold-shell coated silver nanoparticles onto a glass platform for improvement of plasmonic sensors. ACS Appl. Mater. Interfaces, 2013, 5(7): 2392-2399.

[3] SHARMA A K, JHA R, GUPTA B D. Fiber-optic sensors based on surface plasmon resonance: a comprehensive review. IEEE Sens. J., 2007, 7(8): 1118-1129.

[4] FUERTES G, SANCHEZ-MUNOZ O L, PEDRUEZA E, et al. Switchable bactericidal effects from novel silica-coated silver nanoparticles mediated by light irradiation. Langmuir, 2011, 27(6): 2826-2833.

[5] KELLY K L, CORONADO E, ZHAO L L, et al. The optical properties of metal nanoparticles: the influence of size, shape, and dielectric environment. J. Phys. Chem. B, 2003, 107(3): 668-677.

[6] ZHANG L, JIANG C Z, REN F, et al. Optical absorption of nanoclusters by sequentially implanting into $\mathrm{SiO}_{2}$ glass and subsequently annealing in a selected atmosphere. Acta Phys. Sin., 2004, 53(9): 2910-2914.

[7] MOCK J J, BARBIC M, SMITH D R, et al. Shape effects in plasmon resonance of individual colloidal silver nanoparticles. J. Chem. Phys., 2002, 116(15): 6755.

[8] GANEEV R A, BABA M, RYASNYANSKY A I, et al. Characterization of optical and nonlinear optical properties of silver nanoparticles prepared by laser ablation in various liquids. Optics Communications, 2004, 240(4): 437-448.

[9] MA Y, LIN J, ZHU L F, et al. Optical properties of Ag nanoparticle embedded silicate glass prepared by field-assisted diffusion. Appl. Phys. A., 2011, 102(3): 521-525.

[10] POPOK V N, STEPANOV A L, ODZHAEV V B. Synthesis of silver nanoparticles by the ion implantation method and investigation of their optical properties. Journal of Applied Spectroscopy, 2005, 72(2): 229-234.

[11] STEPANOV A L, ZHIKHAREV V A, KHAIBULIN I B. Depth profiles of metal ions implanted in dielectrics at low energies. Phys. Sol. State, 2001, 43(4): 766-771.

[12] ZHANG D C, SHEN Y Y, HUANG Y J, et al. Theoretical study of nanoparticles in insulators fabricated by metal ion implantation. Acta Phys. Sin., 2010, 59(11): 7974-7978.

[13] JOSEPH B, SUCHAND SANDEEP C S, SEKHAR B R, et al. Nonlinear optical properties of $\mathrm{MeV}$ and $\mathrm{keV}$ ion beam synthesized $\mathrm{Ag}$ nanoclusters. Nucl. Instr. and Meth. in Phys. Res. B, 2007， 265(2): 631-636.

[14] STEPANOV A L, GANEEV R A, RYASNYANSKY A I, et al. Non-linear optical properties of metal nanoparticles implanted in silicate glass. Nucl. Instr. and Meth. in Phys. Res. B, 2003, 206: 624-628.

[15] REN F, JIANG C Z, LIU C, et al. Controlling the morphology of $\mathrm{Ag}$ nanoclusters by ion implantation to different doses and subsequent annealing. Physical Review Letters, 2006, 97(16): 165501-1-4.

[16] OLIVER A, CHEANG-WONG J C, ROIZ J, et al. Metallic nanoparticle formation in ion-implanted silica after thermal annealing in reducing or oxidizing atmospheres. Nucl. Instr. and
Meth. in Phys. Res. B, 2002, 191(1): 333-336.

[17] SAHU G, RATH S K, JOSEPH B, et al. Saturation effects observed in high fluence heavy ion implantation at few tens of keV. Vacuum, 2009, 83(5): 836-840.

[18] SHERRY L J, CHANG S H, SCHATZ G C, et al. Localized surface plasmon resonance spectroscopy of single silver nanocubes. Nano Lett., 2005, 5(10): 2034-2038.

[19] HAYNES C L, VAN DUYNE R P. Nanosphere lithography: a versatile nanofabrication tool for studies of size-dependent nanoparticle optics. J. Phys. Chem. B, 2001, 105(24): 5599-5611.

[20] CHAN G H, ZHAO J, HICKS E M, et al. Plasmonic properties of copper nanoparticles fabricated by nanosphere lithography. Nano Lett., 2007, 7(7): 1947-1952.

[21] ROIZ J, OLIVER A, MUNOZ E, et al. Modification of the optical properties of Ag-implanted silica by annealing in two different atmospheres. J. Appl. Phys., 2004, 95(4): 1783-1791.

[22] BI H J, CAI W P, ZHANG L D. Annealing-induced reversible change in optical absorption of Ag nanoparticles. Appl. Phys. Lett., 2002, 81(27): 5222-5224.

[23] STEPANOV A L, POPOK V N. Nanostructuring of silicate glass under low-energy Ag-ion implantation. Surface Science, 2004, 566-568(Part2): 1250-1254.

[24] STEPANOV A L, VALEEV V F, NUZHDIN V I, et al. Specificity of silver nanoparticle synthesis in quartz glass upon low-energy ion implantation. Nanotechnologies in Russia, 2011, 6(7/8): 490-495.

[25] SEO H W, CHEN Q Y, RUSAKOVA I A, et al. Formation of silver nanoparticles in silicon by metal vapor vacuum arc ion implantation. Nucl. Instr. and Meth. in Phys. Res. B, 2012, 292: 50-54.

[26] CHEANG-WONG J C, OLIVER A, RODRIGUEZ-FERNANDEZ $\mathrm{J}$, et al. Relationship between the Ag depth profiles and nanoparticle formation in Ag-implanted silica. J. Phys. Condens. Matter, 2001, 13(45): 10207-10209.

[27] MARQUES C, SILVA R C, WEMANS A, et al. Optical properties tailoring by high fluence implantation of Ag ions on sapphire. Nucl. Instr. and Meth. in Phys. Res. B, 2006, 242(1/2): 104-108.

[28] WANG J, JIA G Y, MU X Y, et al. Quasi-two-dimensional Ag nanoparticle formation in silica by Xe ion irradiation and subsequent Ag ion implantation. Appl. Phys. Lett., 2013, 102(13): 133102-1-4.

[29] POPOK V N, GROMOV A V, NUZHDIN V I, et al. Optical and AFM study of ion-synthesised silver nanoparticles in thin surface layers of $\mathrm{SiO}_{2}$ glass. Journal of Non-Crystalline Solids, 2010, 356(25/26/27): 1258-1261.

[30] XU J X, REN F, FU D J, et al. Effect of thermal annealing on the optical properties of low-energy $\mathrm{Cu}$-implanted silica glass. Physica B: Condensed Matter, 2006, 373(2): 341-345.

[31] REN F, JIANG C Z, LIU C, et al. Interface influence on the surface plasmon resonance of $\mathrm{Ag}$ nanocluster composites. Solid State Communications, 2005, 135(4): 268-272.

[32] PAL S, DE G. Reversible transformations of silver oxide and metallic silver nanoparticles inside $\mathrm{SiO}_{2}$ films. Materials Research Bulletin, 2009, 44(2): 355-359.

[33] BANIJAMALI S, AGHAEI A R, EFTEKHARI YEKTA B. Non-isothermal crystallization kinetics and microstructure of a silver doped calcium aluminophosphate glass. Ceramics International. 2012, 38(3): 2395-2402. 